\title{
Synthesis, azo-hydrazone tautomerism and antitumor screening of $N$-(3-ethoxycarbonyl-4,5,6,7-tetrahydro-benzo[b]thien-2-yl)-2- arylhydrazono-3-oxobutanamide derivatives
}

\author{
Thoraya A. Farghaly* and Zeinab A. Abdallah \\ Department of Chemistry, Faculty of Science, University of Cairo, Giza, Egypt \\ E-mail: thoraya-f@hotmail.com
}

\begin{abstract}
A series of new $N$-(3-ethoxycarbonyl-4,5,6,7-tetrahydrobenzo[ $b]$ thien-2-yl)-2-aryl hydrazono-3oxobutanamide derivatives $4 \mathbf{4}-\mathbf{i}$ were synthesized and the acid dissociation constants $\left(\mathrm{pK}_{\mathrm{a}} \mathrm{s}\right)$ for the series prepared were determined and correlated by the Hammett-type equation using the enhanced substituent constants $\sigma_{\mathrm{x}}{ }^{-}$. The results of such correlation together with the spectral data indicated that the studied compounds exist predominantly in the hydrazone tautomeric form. Some of these derivatives exhibit high antitumor activity.
\end{abstract}

Keywords: Synthesis, coupling, azo-hydrazone tautomerism, benzo[b]thiophene

\section{Introduction}

Azo dyes are versatile compounds that have wide applications in industry and photodynamic therapy as well as photosensitive species in photographic or electrophotographic systems and are dominant organic photoconductive materiels. ${ }^{1}$ Furthermore, thiophenes and their fused derivatives have shown diverse pharmacological activities including antibacterial, ${ }^{2,3}$ antifungal, ${ }^{3}$ immunomodulatory, ${ }^{4}$ antidiabatic, ${ }^{5}$ antiinflammantory, ${ }^{6}$ antiviral activities ${ }^{7,8}$ and anticancer agent. ${ }^{9}$ Therefore, it was thought to be of interest to combine two of the above-mentioned azo dye and thiophene ring together in a molecular framework to give dye with high pharmaceutical potentiality.

\section{Results and Discussion}

The required starting active methylene compound 3 was previously prepared ${ }^{10}$ as a mixture in $15-20 \%$ yield, but in this study we synthesized it by the condensation of compound 1 with ethyl acetoacetate 2 at $160{ }^{\circ} \mathrm{C}$ for $30 \mathrm{~min}$. to give compound $\mathbf{3}$ in $95 \%$ yield (Scheme 1). Compound 3 
can exist in one of two tautomeric structures $\mathbf{3 A}$ or $3 \mathbf{B}$. The IR spectral data seem to be more consistent with the ketone structure $\mathbf{3 A}$, since the IR spectra revealed three bands at 1709, 1682, $1666 \mathrm{~cm}^{-1}$ corresponding to the stretching vibration of the ester, acetyl and amide carbonyl groups, respectively. The low stretching vibrations of ester carbonyl group was due to the hydrogen bonding with the amide NH. In addition, the ${ }^{1} \mathrm{H}$ NMR spectra show the presence of $\mathrm{NH}$ singlet signal at $11.43 \mathrm{ppm}$ and the absence of singlet signal at $5.70 \mathrm{ppm}$ for $=\mathrm{CH}$. These findings indicate that compound $\mathbf{3}$ exist predominantly in the ketone form $\mathbf{3 A}$ (Scheme 1).
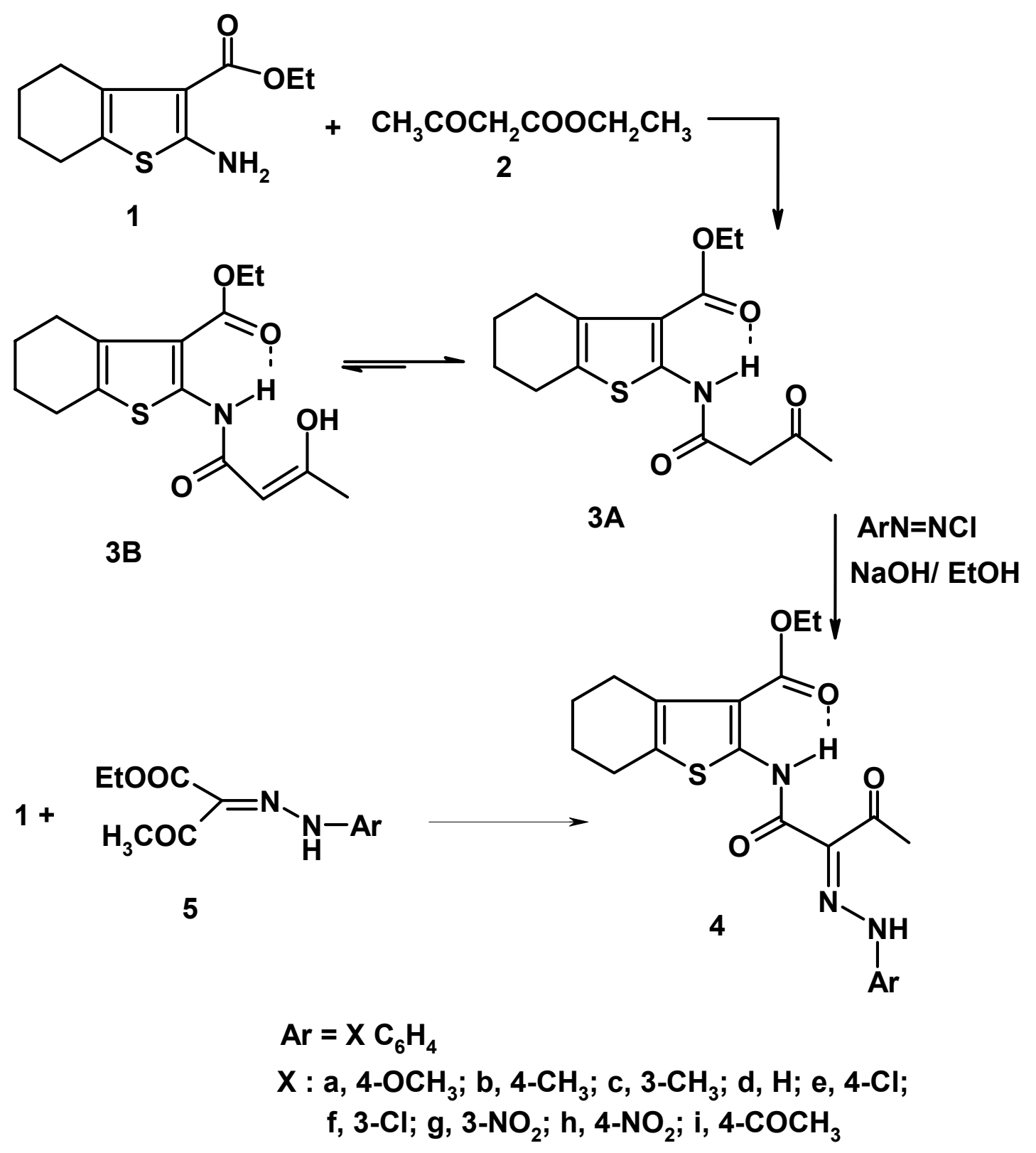

Scheme 1. Synthesis of compound $\mathbf{4 a - i}$. 
Reaction of 3 with diazotized aniline derivatives in aqueous ethanol in the presence of sodium hydroxide afforded the respective arylazo derivatives 4a-i (Scheme 1). The mass spectra of the latter products revealed the molecular ion peaks at the expected $\mathrm{m} / \mathrm{z}$ values with relative intensities varying from 40 to $100 \%$ and their elemental analysis data are consistent with their assigned structure. As shown in Figure 1, compounds 4 can have one or more of three possible tautomeric structures $\mathbf{4 A - C}$ namely, keto-hydrazone $\mathbf{4 A}$, hydroxy-azo $4 \mathrm{~B}$ and $\mathrm{CH}$-azo $\mathbf{4 C}$. Of these forms, structure $\mathbf{4 A}$ seems to be the form of choice as it is consistent with their electronic absorption and ${ }^{1} \mathrm{H}$ NMR spectra. For example, like typical hydrazones, ${ }^{11,12}$ the electronic absorption spectra of $\mathbf{4}$ in dioxane revealed in each case two characteristic absorption bands in the regions 394-411 and 243-281 nm (Table 1). Furthermore, the spectrum of 4d, taken as a typical example of the series studied was recorded in solvents of different polarities. The spectra obtained showed little, if any, shift (Table 1). The small shifts in $\lambda_{\max }$ of $\mathbf{4 d}$ in different solvents are due to solute-solvent interaction. In agreement with this conclusion is the observation that the spectra of arylhydrazones derived from the reaction of quinones with $\mathrm{N}$-alkyl- $\mathrm{N}$ phenylhydrazine, unlike those of $o$ - and $p$-hydroxyazo compounds, are largely independent of the solvent polarity. ${ }^{12}$ This finding, while it excludes the azo tautomeric forms $\mathbf{4 B}$ and $\mathbf{4 C}$, indicates that each of compounds 4 exists in one tautomeric form, namely $\mathbf{4 A}$ (Figure 1). This conclusion was confirmed by the ${ }^{1} \mathrm{H}$ NMR and IR spectra of the studied compounds 4 . Their ${ }^{1} \mathrm{H}$ NMR spectra revealed no signals at $5.3 \mathrm{ppm}$ for $\mathrm{CH}$ of tautomeric form $4 \mathrm{C}$ and their IR spectra revealed three carbonyl absorption bands in regions 1701-1671, 1666-1651 and 1655-1624 $\mathrm{cm}^{-1}$ characteristic for ester, acetyl and amide carbonyl groups, respectively. The observed low frequency of all of these carbonyl groups may be the result of the intramolecular H-bonding.

The assigned structure $\mathbf{4}$ was confirmed by an alternative synthesis of the products $\mathbf{4 a}$ and $\mathbf{4 d}$ as typical examples of the series prepared. Thus, treatment of $\mathbf{1}$ with each of the compounds $\mathbf{5 a}$ and $\mathbf{5 d}$ in dioxan in presence of triethylamine gave the products $4 \mathbf{a}$ and $\mathbf{4 d}$ that proved identical in all respects (m.p., mixed m.p., IR, UV) with those obtained above from the coupling of $\mathbf{3}$ with the respective diazotized anilines (Scheme 1).

To provide further evidence for the tautomeric form $\mathbf{4 A}$ assigned to the studied hydrazone products 4 , their acid dissociation constants, $\mathrm{pK}_{\mathrm{a}} \mathrm{s}$, of the series prepared were determined and their correlation by the Hammett equation was examined. ${ }^{13}$ The acid dissociation constants for the series 4a-i were determined potentiometrically at $27^{\circ} \mathrm{C}$ in $80 \%$ dioxane-water mixture (v/v). In all determinations the ionic strength $\mu$ was kept constant at 0.1 . From the $\mathrm{pH}$ - titrant volume data, the acid dissociation constants of the compounds studied were calculated (see Experimental) and the results are summarized in Table 1. 
<smiles></smiles><smiles></smiles>

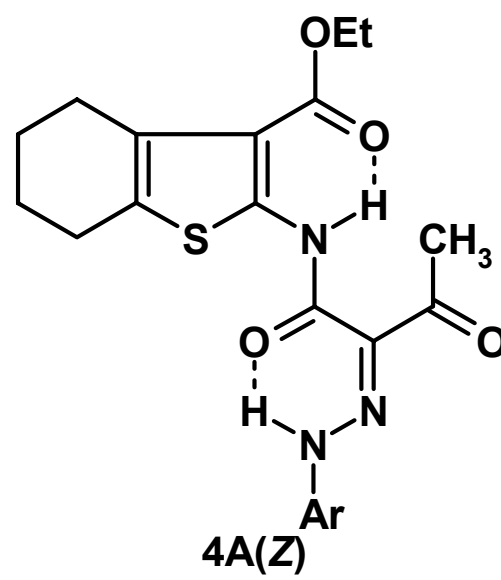

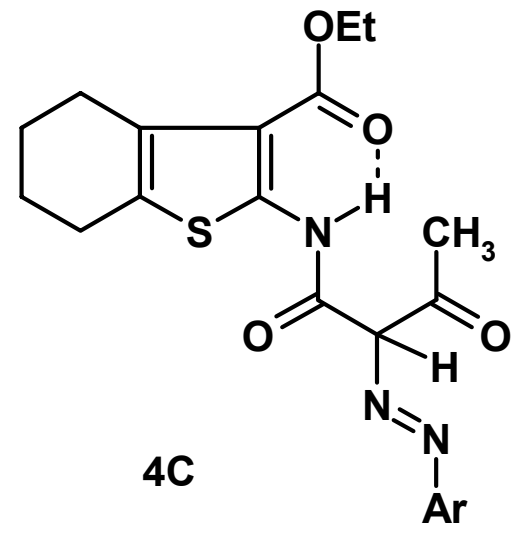

Figure 1. The tautomeric structure of compound 4 A-C

When the $\mathrm{pK}_{\mathrm{a}}$ values were plotted versus Hammett substituent constants $\sigma_{x}{ }^{13}$ all the substituents fall on the correlation line except the substituents with - $\mathrm{R}$ effect, namely the $p$ - $\mathrm{Cl}, p$ $\mathrm{NO}_{2}$ and $p-\mathrm{CH}_{3} \mathrm{CO}$ groups, which are capable of direct interaction with the negatively charged reaction site. However, when the $p K_{a}$ values were plotted versus $\sigma_{x}{ }^{-}$constants, ${ }^{13}$ better correlation was obtained. The Hammett type equation of the regression line obtained is:

$$
p K_{a}=9.05-2.30 \sigma_{x}^{-} \quad ; r=0.999 ; s= \pm 0.04
$$

where $\boldsymbol{r}$ is the correlation coefficient and $\boldsymbol{s}$ is the standard deviation.

This excellent linear correlation indicates that the parameter $\boldsymbol{r}^{-}$in the Humffray-Ryan equation ${ }^{14}$ :

$$
p K_{a}=p K_{a}{ }^{\mathrm{o}}+\rho\left\{\sigma_{x}+r^{-}\left(\sigma_{x}^{-}-\sigma_{x}\right)\right\},
$$

which gives the contribution of the resonance effect of the substituent varied, is close to unity for the series 4a-i studied.

The foregoing linear correlation between $\mathrm{pK}_{\mathrm{a}}$ values and $\sigma_{x}{ }^{-}$constants and the values of $\rho$ and $r$ found provided further evidence that the studied compounds 4 exist predominantly in the 
hydrazone form 4A. This is because the values of $\rho(2.30)$ and $r^{-}=1.00$ are similar to those reported for ionization of phenols $\left(\rho=2.67 ; r^{-}=1.00\right)$ and anilinium ions $\left(\rho=2.77 ; r^{-}=1.00\right)$ in $50 \%$ ethanol-water mixture. ${ }^{15,16}$ This finding indicates that the negative charge on the anion formed by deprotonation of $\mathbf{4}$ is largely localized on the $\mathrm{N}$-atom adjacent to the benzene bearing the substituent. This anion will take up a proton on the $\mathrm{N}$-atom bearing the negative charge to give $\mathbf{4 A}$ which is also stabilized by hydrogen bonding. Furthermore, if either form $\mathbf{4 B}$ or $\mathbf{4 C}$ were the predominant form for the studied compounds, the $\rho$ values would be less than 2.0 and will be similar to that reported for ionization of 2 -arylazophenols $\left(\rho=1.223 ; r^{-}=0.286\right){ }^{15}$ Since the bridge between the substituent and the deprotonation reaction site in either form $\mathbf{4 B}$ or 4C is longer than in $\mathbf{4 A}$. Thus, it is not unreasonable to conclude that the observed linear correlation of the acid dissociation constants with the Hammett equation indicates that the hydrazone tautomeric form $\mathbf{4 A}$ prevails under the conditions of the $p K_{a}$ measurement. Compound $\mathbf{4 A}$ can exist in the $E$-structure or the $Z$-structure, the E-structure of hydrazone 4A was preferred than the $Z$-structure ${ }^{17-19}$ in which the acetyl carbonyl rather than the amide carbonyl was involved in the H-bonded system.

Table 1. UV Spectral data and acid dissociation constants ${ }^{\mathrm{a}}$ of compounds $\mathbf{4 a - i}$ in dioxane

\begin{tabular}{cccc}
\hline Compd. no. & $\lambda_{\max }(\log \varepsilon)$ & $\mathrm{pKa}( \pm \mathrm{s})$ & $\sigma^{-} \mathrm{x}$ \\
\hline $\mathbf{4 a}$ & $411(4.76), 255(4.57)$ & $9.65(0.02)$ & -0.27 \\
$\mathbf{4 b}$ & $401(4.86), 245(5.30)$ & $9.43(0.01)$ & -0.17 \\
$\mathbf{4 c}$ & $396(4.89), 243(5.30)$ & $9.20(0.01)$ & -0.07 \\
$\mathbf{4 d}^{\mathrm{b})}$ & $396(4.61), 281(3.91)$ & $9.08(0.03)$ & 0.0 \\
$\mathbf{4 e}$ & $400(4.60), 252(4.49)$ & $8.55(0.02)$ & 0.23 \\
$\mathbf{4 f}$ & $394(4.68), 244(5.30)$ & $8.18(0.03)$ & 0.37 \\
$\mathbf{4 g}$ & $395(4.82), 244(5.30)$ & $7.43(0.01)$ & 0.71 \\
$\mathbf{4 h}$ & $411(4.55), 243(5.30)$ & $6.10(0.03)$ & 1.28 \\
$\mathbf{4 i}$ & $395(4.52), 246(4.90)$ & $7.57(0.07)$ & 0.68 \\
\hline
\end{tabular}

${ }^{\mathrm{a}} \mathrm{pK}_{\mathrm{a}}$ in dioxane-water $(4: 1 \mathrm{v} / \mathrm{v})$ solution at $27^{\circ} \mathrm{C}$ and $\mu=0.10 .{ }^{\mathrm{b}}$ Solvent $\lambda_{\max }(\log \varepsilon)$ : acetic acid 397 (4.20), 235 (4.65); ethanol 397 (4.78), 244 (4.75); DMF 396 (4.90), 258 (5.30); DMSO 399 (4.87); 251 (5.30).

\section{Pharmacology}

\section{Antitumor screening test}

The cytotoxic effects of the synthesized compounds $\mathbf{3}$ and $\mathbf{4}$ at three different concentrations of each, and doxorubicin (Adriablastina), as a reference drug, against Ehrlich Ascites Carcinoma tumor cells (EAC) were evaluated in vitro. 
As shown in Table 2, the results of antitumor activity screening of the studied compounds indicate that compounds $3, \mathbf{4 b}, \mathbf{4 e}, \mathbf{4 f}$ and $\mathbf{4 g}$ have high degree of antitumor activities compaired to the positive control toward Ehrlich Ascites Carcinoma tumor cells (in vitro). The other derivatives of compounds $\mathbf{4}$ have moderate or low antitumor activities toward Ehrlich Ascites Carcinoma tumor cells (in vitro) in relation with the reference drug doxorubicin (Table 2).

Table 2. In vitro cytotoxic activity of compounds $\mathbf{3 , 4}$ and doxorubicin against (EAC)

\begin{tabular}{cccc}
\hline \multirow{2}{*}{$\begin{array}{c}\text { Compound } \\
\text { No. }\end{array}$} & \multicolumn{3}{c}{$\begin{array}{c}\text { Non-viable cells }(\%) \\
\text { concentration }(\mu \mathrm{g} / \mathrm{ml})\end{array}$} \\
\cline { 2 - 4 } & 100 & 50 & 25 \\
\hline $\mathbf{3}$ & 70 & 30 & 10 \\
$\mathbf{4 a}$ & 27 & 12 & 0 \\
$\mathbf{4 b}$ & 52 & 20 & 10 \\
$\mathbf{4 c}$ & 30 & 14 & 7 \\
$\mathbf{4 d}$ & 36 & 17 & 8 \\
$\mathbf{4 e}$ & 50 & 24 & 11 \\
$\mathbf{4 f}$ & 48 & 22 & 10 \\
$\mathbf{4 g}$ & 70 & 34 & 14 \\
$\mathbf{4 h}$ & 44 & 21 & 9 \\
$\mathbf{4 i}$ & 22 & 9 & 0 \\
Cell control & $100 \%$ & $100 \%$ & $100 \%$ \\
doxorubicin & $25 \%$ & $13 \%$ & $6 \%$ \\
\hline
\end{tabular}

In conclusion, a simple method for the preparation of compound 3 in $95 \%$ yield and their coupling reaction to give new hydrazone derivatives $\mathbf{4 a - i}$ is described. The structures of the newly synthesized compounds 4 a-i were confirmed by spectral and elemental analyses data and the correlation with Hammett equation. These new compounds have been evaluated for the antitumor activity, compounds $3, \mathbf{4 b}, \mathbf{4 e}, \mathbf{4 f}$ and $\mathbf{4 g}$ have high antitumor activity against Ehrlich Ascites Carcinoma cells. The other derivatives in series $\mathbf{4}$ exhibit moderate or low activity.

\section{Experimental Section}

General Procedures. Melting points were determined on a Gallenkamp apparatus and are uncorrected. IR spectra were recorded in potassium bromide using Perkin Elmer FTIR 1650 and Pye-Unicam SP300 infrared spectrophotometers. ${ }^{1} \mathrm{H}$ NMR spectra were recorded in deuterated dimethylsulfoxide using a Varian Gemini 300 NMR spectrometer. Mass spectra were recorded on a GCMS-QP 1000 EX Shimadzu and GCMS 5988-A HP spectrometers. Electronic absorption spectra were recorded on Perkin-Elmer Lambada 40 spectrophotometer. Elemental analyses were 
carried out at the Microanalytical Laboratory of Cairo University, Giza, Egypt. Antitumor screening was done in the National Institute of Cancer, Cairo, Egypt.

Reaction of ethyl acetoacetate with ethyl 2-amino-4,5,6,7-tetrahydrobenzo $[b]$ thiophene-3carboxylate (1). Ethyl acetoacetate $2(2.93 \mathrm{~g}, 0.023 \mathrm{~mole})$ was heated in conical flask at $100^{\circ} \mathrm{C}$, then ethyl 2-amino-4,5,6,7-tetrahydrobenzo[b]thiophene-3-carboxylate 1 (3.38 g, $0.015 \mathrm{~mol})$ was added portionwise with stirring. The temperature was then raised up to $150-160{ }^{\circ} \mathrm{C}$ for $20 \mathrm{~min}$. the reaction mixture was left to cool, the solid product was filtered off, washed with ethanol and recrystallized from ethanol to give compound $\mathbf{3}$ as yellow crystals $\mathrm{mp} .80{ }^{\circ} \mathrm{C}$ (lit. mp. $90-92{ }^{\circ} \mathrm{C}$ in $15-20 \%$ yield $^{10}$ ); yield $4.40 \mathrm{~g}(95 \%)$. IR $v / \mathrm{cm}^{-1}: 3163,2981,2939,2843,1709,1682,1666$ $\mathrm{cm}^{-1} .{ }^{1} \mathrm{H}$ NMR (DMSO-d $)$ ): $\delta / \mathrm{ppm}: 1.31\left(\mathrm{t}, J=7.0 \mathrm{~Hz}, 3 \mathrm{H}, \mathrm{CH}_{3}\right), 1.71\left(\mathrm{~m}, 4 \mathrm{H}, 2 \mathrm{CH}_{2}\right), 2.20(\mathrm{~s}$, $\left.3 \mathrm{H}, \mathrm{COCH}_{3}\right), 2.59-2.70\left(\mathrm{~m}, 4 \mathrm{H}, 2 \mathrm{CH}_{2}\right), 3.85\left(\mathrm{~s}, 2 \mathrm{H}, \mathrm{CH}_{2}\right), 4.28$ (q, J=7.0 Hz, 2H, $\left.\mathrm{CH}_{2}\right), 11.43$ (s, 1H, NH). MS m/z (\%) $311\left(\mathrm{M}^{+}+2,8\right), 310\left(\mathrm{M}^{+}+1,24\right), 309\left(\mathrm{M}^{+}, 57\right), 225$ (64), 179 (100), 151(24), 91 (7). Anal. Calcd. For $\mathrm{C}_{15} \mathrm{H}_{19} \mathrm{NO}_{4} \mathrm{~S}$ (309.38) C, 58.23; H,6.19; N,4.53\%. Found: C, $58.16 ; \mathrm{H}, 6.03 ; \mathrm{N}, 4.32 \%$.

\section{Preparation of compounds (4a-i)}

Method A. To a stirred solution of compound 3 ( $3.09 \mathrm{~g}, 10 \mathrm{mmol})$ in ethanol $(50 \mathrm{~mL})$ was added sodium hydroxide $(0.4 \mathrm{~g}, 10 \mathrm{mmol})$ and the mixture was cooled in an ice bath to $0-5{ }^{\circ} \mathrm{C}$. To the resulting solution, while being stirred, was added dropwise over a period of $20 \mathrm{~min}$., a solution of the appropriate arenediazonium chloride, prepared as usual by diazotizing the respective aniline $(10 \mathrm{mmol})$ in hydrochloric acid $(6 \mathrm{M}, 6 \mathrm{~mL})$ with sodium nitrite $(1 \mathrm{M}, 10 \mathrm{~mL})$. The whole mixture was then left overnight in a refrigerator. The precipitate solid was collected, washed with water and finally crystallized from the appropriate solvent to give the respective hydrazones (4a-i)

Method B. A mixture of 1 ( 5 mmole) with ethyl 2-arylhydrazono-3-oxobutanoate 5a,d $(5 \mathrm{mmole})$ in dioxan $(30 \mathrm{~mL})$ and triethyl amine $(0.35 \mathrm{~mL})$ was heated to reflux for $5 \mathrm{~h}$, then cooled. The solid formed was collected by filtration and crystallized from the appropriated solvent to give the corresponding compounds $\mathbf{4 a}$ and $\mathbf{4 d}$ which was found identical in all respects with that produced by Method A.

\section{$\mathrm{N}$-(3-Ethoxycarbonyl-4,5,6,7-tetrahydrobenzo[b]thien-2-yl)-2-(4-methoxyphenyl}

hydrazono)-3-oxobutanamide (4a). Orange solid, m.p. $216^{\circ} \mathrm{C}$ (Dioxane); yield $3.77 \mathrm{~g} \mathrm{(85 \% ).}$ IR $v / \mathrm{cm}^{-1}: 3409,3070,2927,2835,1681,1651,1629 \mathrm{~cm}^{-1} .{ }^{1} \mathrm{H}$ NMR (DMSO-d $\left.\mathrm{d}_{6}\right): \delta / p p m: 1.34(\mathrm{t}$, $\left.J=7.0 \mathrm{~Hz}, 3 \mathrm{H}, \mathrm{CH}_{3}\right), 1.73\left(\mathrm{~m}, 4 \mathrm{H}, 2 \mathrm{CH}_{2}\right), 2.49\left(\mathrm{~s}, 3 \mathrm{H}, \mathrm{CH}_{3}\right), 2.51-2.73\left(\mathrm{~m}, 4 \mathrm{H}, 2 \mathrm{CH}_{2}\right), 3.78(\mathrm{~s}$, $\left.3 \mathrm{H}, \mathrm{CH}_{3}\right), 4.35\left(\mathrm{q}, J=7.0 \mathrm{~Hz}, 2 \mathrm{H}, \mathrm{CH}_{2}\right), 7.01$ (d, $\left.J=8.0 \mathrm{~Hz}, 2 \mathrm{H}, \mathrm{Ar}-\mathrm{H}\right), 7.54(\mathrm{~d}, J=8.0 \mathrm{~Hz}, 2 \mathrm{H}$, Ar-H), 13.33 (s, 1H, NH), 14.00 (s, 1H, NH). MS m/z (\%) $444\left(\mathrm{M}^{+}+1,17\right), 443\left(\mathrm{M}^{+}, 45\right), 226$ (11), 225 (100), 179 (33), 122 (32), 107 (11), 77 (7). Anal. Calcd. For $\mathrm{C}_{22} \mathrm{H}_{25} \mathrm{~N}_{3} \mathrm{O}_{5} \mathrm{~S}$ (443.52) C, 59.58; H, 5.68; N, 9.47\%. Found: C, 59.26; H, 5.80; N, $9.56 \%$.

$\mathrm{N}$-(3-Ethoxycarbonyl-4,5,6,7-tetrahydrobenzo $[\boldsymbol{b}]$ thien-2-yl)-2-(4-methylphenyl hydrazono) -3-oxobutanamide (4b). Orange crystals, m.p. $202{ }^{\circ} \mathrm{C}$ (Dioxane); yield 3.84 g (90\%). IR v/cm ${ }^{-1}$ : 
3425, 3078, 2931, 2854, 1682, 1655, $1616 \mathrm{~cm}^{-1} .{ }^{1} \mathrm{H}$ NMR (DMSO-d 6 ): $\delta / p p m: 1.34$ (t, $J=7.0$ $\left.\mathrm{Hz}, 3 \mathrm{H}, \mathrm{CH}_{3}\right), 1.73\left(\mathrm{~m}, 4 \mathrm{H}, 2 \mathrm{CH}_{2}\right), 2.31\left(\mathrm{~s}, 3 \mathrm{H}, \mathrm{CH}_{3}\right), 2.49\left(\mathrm{~s}, 3 \mathrm{H}, \mathrm{CH}_{3}\right), 2.60-2.73(\mathrm{~m}, 4 \mathrm{H}$, $\left.2 \mathrm{CH}_{2}\right), 4.36$ (q, $\left.J=7.0 \mathrm{~Hz}, 2 \mathrm{H}, \mathrm{CH}_{2}\right), 7.24(\mathrm{~d}, J=9.0 \mathrm{~Hz}, 2 \mathrm{H}, \mathrm{Ar}-\mathrm{H}), 7.45(\mathrm{~d}, J=9.0 \mathrm{~Hz}, 2 \mathrm{H}$, Ar-H), 13.32 (s, 1H, NH), 13.98 (s, 1H, NH). MS m/z (\%) $428\left(\mathrm{M}^{+}+1,21\right), 427\left(\mathrm{M}^{+}, 49\right), 225$ (100), 179 (51), 151 (15), 106 (32), 91 (25), 77 (19); Anal. Calcd. For $\mathrm{C}_{22} \mathrm{H}_{25} \mathrm{~N}_{3} \mathrm{O}_{4} \mathrm{~S}$ (427.52) C, $61.81 ; \mathrm{H}, 5.89 ; \mathrm{N}, 9.83 \%$. Found: C, 61.74; H, 5.66; N, $9.78 \%$.

$\mathrm{N}$-(3-Ethoxycarbonyl-4,5,6,7-tetrahydrobenzo[b]thien-2-yl)-2-(3-methylphenyl hydrazono)3-oxobutanamide (4c). Orange solid, m.p. $180{ }^{\circ} \mathrm{C}$ (Dioxane); yield $3.50 \mathrm{~g}(82 \%)$. IR v/ $\mathrm{cm}^{-1}$ : 3454, 3129, 2941, 2861, 1682, 1666, $1655 \mathrm{~cm}^{-1} .{ }^{1} \mathrm{H}$ NMR (DMSO-d $\left.\mathrm{d}_{6}\right): \delta / p p m: 1.31(\mathrm{t}, J=7.0$ $\left.\mathrm{Hz}, 3 \mathrm{H}, \mathrm{CH}_{3}\right), 1.70\left(\mathrm{~m}, 4 \mathrm{H}, 2 \mathrm{CH}_{2}\right), 2.33\left(\mathrm{~s}, 3 \mathrm{H}, \mathrm{CH}_{3}\right), 2.50\left(\mathrm{~s}, 3 \mathrm{H}, \mathrm{CH}_{3}\right), 2.51-2.68(\mathrm{~m}, 4 \mathrm{H}$, $2 \mathrm{CH}_{2}$ ), 4.33 (q, $\left.J=7.0 \mathrm{~Hz}, 2 \mathrm{H}, \mathrm{CH}_{2}\right), 7.00-7.30(\mathrm{~m}, 4 \mathrm{H}, \mathrm{Ar}-\mathrm{H}), 13.26$ (s, $\left.1 \mathrm{H}, \mathrm{NH}\right), 13.93(\mathrm{~s}, 1 \mathrm{H}$, $\mathrm{NH})$. MS m/z (\%) $428\left(\mathrm{M}^{+}+1,31\right), 427\left(\mathrm{M}^{+}, 45\right), 225$ (100), 178 (16), 106 (23), 91 (26), 77 (17). Anal. Calcd. For $\mathrm{C}_{22} \mathrm{H}_{25} \mathrm{~N}_{3} \mathrm{O}_{4} \mathrm{~S}$ (427.52) C, 61.81; H, 5.89; N, 9.83\%. Found: C, 61.63; H, 5.44; $\mathrm{N}, 9.58 \%$.

$\mathrm{N}$-(3-Ethoxycarbonyl-4,5,6,7-tetrahydrobenzo[b]thien-2-yl)-2-phenylhydrazono-3-

oxobutanamide (4d). Orange crystals, m.p. $208{ }^{\circ} \mathrm{C}$ (Dioxane/Ethanol); yield $3.80 \mathrm{~g}(92 \%)$. IR $v / \mathrm{cm}^{-1}: 3429,3074,2928,2851,1681,1659,1624 \mathrm{~cm}^{-1} .{ }^{1} \mathrm{H}$ NMR (DMSO-d 6 ): $\delta / p p m: 1.34$ (t, $J=$ $\left.7.0 \mathrm{~Hz}, 3 \mathrm{H}, \mathrm{CH}_{3}\right), 1.72\left(\mathrm{~m}, 4 \mathrm{H}, 2 \mathrm{CH}_{2}\right), 2.49\left(\mathrm{~s}, 3 \mathrm{H}, \mathrm{CH}_{3}\right), 2.60-2.72\left(\mathrm{~m}, 4 \mathrm{H}, 2 \mathrm{CH}_{2}\right), 4.35(\mathrm{q}, J=$ $\left.7.0 \mathrm{~Hz}, 2 \mathrm{H}, \mathrm{CH}_{2}\right), 7.21-7.57$ (m, 5H, Ar-H), 13.30 (s, 1H, NH), 13.94 (s, 1H, NH). MS m/z (\%) $414\left(\mathrm{M}^{+}+1,50\right), 413\left(\mathrm{M}^{+}, 64\right), 225$ (100), 224 (12), 179 (66), 151 (15), 92 (24), 91 (11), 77 (22). Anal. Calcd. For $\mathrm{C}_{21} \mathrm{H}_{23} \mathrm{~N}_{3} \mathrm{O}_{4} \mathrm{~S}$ (413.49) C, 61.00; H, 5.61; N, 10.16\%. Found: C, 60.98; H, 5.40; $\mathrm{N}, 10.00 \%$.

$\mathrm{N}$-(3-Ethoxycarbonyl-4,5,6,7-tetrahydrobenzo[b]thien-2-yl)-2-(4-chlorophenyl hydrazono)3-oxobutanamide (4e). Orange solid, m.p. $214-218{ }^{\circ} \mathrm{C}$ (Dioxane); yield $3.80 \mathrm{~g}(89 \%)$. IR $v / \mathrm{cm}^{-1}$ : 3422, 3074, 2928, 2850, 1681, 1658, $1630 \mathrm{~cm}^{-1} .{ }^{1} \mathrm{H}$ NMR (DMSO-d $\left.{ }_{6}\right): \delta / p p m: 1.34$ (t, $J=7.0$ $\left.\mathrm{Hz}, 3 \mathrm{H}, \mathrm{CH}_{3}\right), 1.72\left(\mathrm{~m}, 4 \mathrm{H}, 2 \mathrm{CH}_{2}\right), 2.50\left(\mathrm{~s}, 3 \mathrm{H}, \mathrm{CH}_{3}\right), 2.61\left(\mathrm{~m}, 2 \mathrm{H}, \mathrm{CH}_{2}\right), 2.73\left(\mathrm{~m}, 2 \mathrm{H}, \mathrm{CH}_{2}\right)$, 4.36 (q, $\left.J=7.0 \mathrm{~Hz}, 2 \mathrm{H}, \mathrm{CH}_{2}\right), 7.47$ (d, $\left.J=9.0 \mathrm{~Hz}, 2 \mathrm{H}, \mathrm{Ar}-\mathrm{H}\right), 7.60$ (d, $\left.J=9.0 \mathrm{~Hz}, 2 \mathrm{H}, \mathrm{Ar}-\mathrm{H}\right)$, 13.30 (s, 1H, NH), 13.88 (s, 1H, NH). MS m/z (\%) $449\left(\mathrm{M}^{+}+2,30\right), 447\left(\mathrm{M}^{+}, 40\right), 226(20), 225$ (100), 179 (53), 178 (14), 126 (17), 111 (13), 91 (16), 77 (9); Anal. Calcd. For $\mathrm{C}_{21} \mathrm{H}_{22} \mathrm{ClN}_{3} \mathrm{O}_{4} \mathrm{~S}$ (447.94): C, 56.31; H, 4.95; N, 9.38\%. Found: C, 56.30; H, 4.86; N, $9.24 \%$.

$\mathrm{N}$-(3-Ethoxycarbonyl-4,5,6,7-tetrahydrobenzo[b]thien-2-yl)-2-(3-chlorophenyl hydrazono)3-oxobutanamide (4f). Orange solid, m.p. $205{ }^{\circ} \mathrm{C}$ (Dioxane); yield $3.53 \mathrm{~g}(79 \%)$. IR $v / \mathrm{cm}^{-1}$ : 3429, 3105, 2977, 2931, 2854, 1680, 1654, $1624 \mathrm{~cm}^{-1} .{ }^{1} \mathrm{H}$ NMR (DMSO-d 6 ): $\delta / p p m: 1.34$ (t, $J=$ $\left.7.0 \mathrm{~Hz}, 3 \mathrm{H}, \mathrm{CH}_{3}\right), 1.70\left(\mathrm{~m}, 4 \mathrm{H}, 2 \mathrm{CH}_{2}\right), 2.48\left(\mathrm{~s}, 3 \mathrm{H}, \mathrm{CH}_{3}\right), 2.51-2.69\left(\mathrm{~m}, 4 \mathrm{H}, 2 \mathrm{CH}_{2}\right), 4.34$ (q, $J=$ $\left.7.0 \mathrm{~Hz}, 2 \mathrm{H}, \mathrm{CH}_{2}\right), 7.20-7.59$ (m, 4H, Ar-H), 13.24 (s, 1H, NH), 13.79 (s, 1H, NH). MS m/z (\%) $449\left(\mathrm{M}^{+}+2,20\right), 448\left(\mathrm{M}^{+}+1,15\right), 447\left(\mathrm{M}^{+}, 61\right), 226$ (24), 225 (100), 180 (13), 179 (67), 151 (17), 126 (22), 111 (12), 91 (8). Anal. Calcd. For $\mathrm{C}_{21} \mathrm{H}_{22} \mathrm{ClN}_{3} \mathrm{O}_{4} \mathrm{~S}$ (447.94): C, 56.31; H, 4.95; N, 9.38\%. Found: C, $56.20 ; \mathrm{H}, 4.71 ; \mathrm{N}, 9.09 \%$.

$\mathrm{N}$-(3-Ethoxycarbonyl-4,5,6,7-tetrahydrobenzo[b]thien-2-yl)-2-(3-nitrophenyl hydrazono)-3oxobutanamide (4g). Dark yellow solid, m.p. $220^{\circ} \mathrm{C}$ (Dioxane); yield $3.57 \mathrm{~g}(78 \%)$. IR $v / \mathrm{cm}^{-1}$ : 
3409, 3066, 2981, 2927, 2881, 1680, 1662, $1624 \mathrm{~cm}^{-1} .{ }^{1} \mathrm{H}$ NMR (DMSO-d $): \delta / p p m: 1.34(\mathrm{t}, J=$ $\left.7.0 \mathrm{~Hz}, 3 \mathrm{H}, \mathrm{CH}_{3}\right), 1.70\left(\mathrm{~m}, 4 \mathrm{H}, 2 \mathrm{CH}_{2}\right), 2.49\left(\mathrm{~s}, 3 \mathrm{H}, \mathrm{CH}_{3}\right), 2.57-2.66\left(\mathrm{~m}, 4 \mathrm{H}, 2 \mathrm{CH}_{2}\right), 4.33(\mathrm{q}, J=$ $\left.7.0 \mathrm{~Hz}, 2 \mathrm{H}, \mathrm{CH}_{2}\right), 7.65-7.8 .32$ (m, 4H, Ar-H), 13.19 (s, 1H, NH), 13.82 (s, 1H, NH). MS m/z (\%) 458 (M+, 100), 226 (14), 225 (77), 179 (53), 151 (25), 127 (14), 125 (28), 91 (11), 77 (10). Anal. Calcd. For $\mathrm{C}_{21} \mathrm{H}_{22} \mathrm{~N}_{4} \mathrm{O}_{6} \mathrm{~S}$ (458.49): C, 55.01; H, 4.84; N, 12.22\%. Found: C, 54.91; H, 4.60; N, $12.06 \%$.

$\mathrm{N}$-(3-Ethoxycarbonyl-4,5,6,7-tetrahydrobenzo[b]thien-2-yl)-2-(4-nitrophenyl hydrazono)-3oxobutanamide (4h). Orange solid, m.p. $240{ }^{\circ} \mathrm{C}$ (DMF); yield $4.08 \mathrm{~g}(89 \%) . \mathrm{IR} v / \mathrm{cm}^{-1}: 3406$, $3058,2981,2931,2835,1701,1670,1654, \mathrm{~cm}^{-1} .{ }^{1} \mathrm{H}$ NMR (DMSO-d 6 ): $\delta / p p m: 1.34(\mathrm{t}, J=7.0$ $\left.\mathrm{Hz}, 3 \mathrm{H}, \mathrm{CH}_{3}\right), 1.73\left(\mathrm{~m}, 4 \mathrm{H}, 2 \mathrm{CH}_{2}\right), 2.49\left(\mathrm{~s}, 3 \mathrm{H}, \mathrm{CH}_{3}\right), 2.54-2.72\left(\mathrm{~m}, 4 \mathrm{H}, 2 \mathrm{CH}_{2}\right), 4.35(\mathrm{q}, J=7.0$ $\left.\mathrm{Hz}, 2 \mathrm{H}, \mathrm{CH}_{2}\right), 7.73$ (d, J=9.0 Hz, 2H, Ar-H), 8.24 (d, $\left.J=9.0 \mathrm{~Hz}, 2 \mathrm{H}, \mathrm{Ar}-\mathrm{H}\right), 13.23$ (s, 1H, NH), 13.87 (s, 1H, NH). MS m/z (\%) $459\left(\mathrm{M}^{+}+1,24\right), 458\left(\mathrm{M}^{+}, 60\right), 225$ (100), 206 (18), 180 (23), 179 (69), 178 (20), 151 (22), 150 (18), 137 (11), 77 (11). Anal. Calcd. For $\mathrm{C}_{21} \mathrm{H}_{22} \mathrm{~N}_{4} \mathrm{O}_{6} \mathrm{~S}$ (458.49): C, 55.01; H, 4.84; N, 12.22\%. Found: C, 54.93; H, 4.59; N, $12.15 \%$.

$\boldsymbol{N}$-(3-Ethoxycarbonyl-4,5,6,7-tetrahydrobenzo $[\boldsymbol{b}]$ thien-2-yl)-2-(4-acetylphenyl hydrazono)3-oxobutanamide (4i). Red solid, m.p. 236-237 ${ }^{\circ} \mathrm{C}$ (Dioxane); yield $3.91 \mathrm{~g}(89 \%) . \mathrm{IR} \mathrm{v/ \textrm {cm } ^ { - 1 }}$ 3448, 3087, 2981, 2939, 2864, 1682, 1671, 1635, $1624 \mathrm{~cm}^{-1} .{ }^{1} \mathrm{H}$ NMR (DMSO-d ${ }_{6}$ ): $\delta / p p m: 1.33$ $\left(\mathrm{t}, J=7.0 \mathrm{~Hz}, 3 \mathrm{H}, \mathrm{CH}_{3}\right), 1.75\left(\mathrm{~m}, 4 \mathrm{H}, 2 \mathrm{CH}_{2}\right), 2.49\left(\mathrm{~s}, 3 \mathrm{H}, \mathrm{CH}_{3}\right), 2.56\left(\mathrm{~s}, 3 \mathrm{H}, \mathrm{CH}_{3}\right), 2.65-2.76$ $\left(\mathrm{m}, 4 \mathrm{H}, 2 \mathrm{CH}_{2}\right), 4.38\left(\mathrm{q}, J=7.0 \mathrm{~Hz}, 2 \mathrm{H}, \mathrm{CH}_{2}\right), 7.80(\mathrm{~d}, J=9.0 \mathrm{~Hz}, 2 \mathrm{H}, \mathrm{Ar}-\mathrm{H}), 8.28(\mathrm{~d}, J=9.0$ Hz, 2H, Ar-H), 13.29 (s, 1H, NH), 13.89 (s, 1H, NH). MS m/z (\%) $456\left(\mathrm{M}^{+}+1,6\right), 455\left(\mathrm{M}^{+}, 60\right)$, 321 (25), 245 (16), 226 (16), 204 (18), 179 (16), 178 (18), 151 (14), 150 (11), 120 (48), 119 (86), 106 (33), 91 (100), 77 (43). Anal. Calcd. For $\mathrm{C}_{23} \mathrm{H}_{25} \mathrm{~N}_{3} \mathrm{O}_{5} \mathrm{~S}$ (455.53): C, 60.64; H, 5.53; N, 9.22\%. Found: C, 60.45; H, 5.41; N, $9.05 \%$.

\section{$\mathbf{p K}_{\mathrm{a}}$ Determination of Compounds (4a-i)}

The acid dissociation constants of compounds 4 were determined potentiometrically in $80 \%$ dioxan-water mixture at $27 \pm 0.1{ }^{\circ} \mathrm{C}$ and ionic strength $\left(\mathrm{KNO}_{3}\right) \mu 0.1$. A Meetrohm 686 titroprocessor equipped with 665 Dosimat was used. The electrode and the titroprocessor were calibrated with standard Beckman buffer solutions of $\mathrm{pH} 4.01$ and 7.00. The $\mathrm{pH}$ meter reading B recorded in dioxane-water solution was converted to hydrogen ion concentration $\left[\mathrm{H}^{+}\right]$by means of the relation of van Uitert and $\mathrm{Hass}^{20}$ namely:

$-\log \left[\mathrm{H}^{+}\right]=\mathrm{B}+\log \mathrm{U}_{\mathrm{H}}$

Where $\log \mathrm{U}_{\mathrm{H}}$ is the correction factor for the solvent composition and ionic strength used for which $B$ is read. The value of $\log \mathrm{U}_{\mathrm{H}}$ was found to be 0.48 . A carbonate-free sodium hydroxide titrant was prepared and standardized against potassium hydrogen phthalate solution.

The experimental procedure followed in the determination of $p K_{a}$ values and their calculations, by the method of least squares, from the titrant volume-pH data using the relation: $p K_{a}=\mathrm{pH}_{\mathrm{i}}-$ $\log \mathrm{V}_{\mathrm{i}} /\left(\mathrm{V}_{\mathrm{e}}-\mathrm{V}_{\mathrm{i}}\right)$, is similar to that previously described. ${ }^{21}$ In this equation, $\mathrm{pH}_{\mathrm{i}}$ is the corrected $\mathrm{pH}$ value of the solution when the volume of the added titrant is $\mathrm{V}_{\mathrm{i}}$ and $\mathrm{V}_{\mathrm{e}}$ is the volume of the titrant at the equivalence point. The calculations of the $p K_{a}$ values were carried out using 
computer program MINIQUAD-75. ${ }^{22}$ The $p K_{a}$ values obtained were reproducible to within \pm $0.02 p K_{a}$ unit. The results are summarized in Table 1.

Cytotoxic activity against Ehrlich Ascites Carcinoma tumor cell (EAC) in vitro ${ }^{23}$ : Ehrlich Ascites Carcinoma cell (EAC) used in this study was kindly supplied by National Institute of Cancer in Netherland since 1952 and mentained in Swiss albino mice by serial I P transplantation, RPMI 1640 medium (Sigma), Ehrlich Ascites Carcinoma cell (EAC) suspension $\left(2.5 \times 10^{5} \mathrm{cells} / \mathrm{ml}\right)$, and Trypan blue dye were used. A stock solution was prepared by dissolving one gram of the dye in distilled water $(100 \mathrm{ml})$. The working solution was then prepared by diluting $(1 \mathrm{ml})$ of the stock solution with $9 \mathrm{ml}$ of distilled water. The stain was then used for staining the dead EAC cells.

A set of sterile test tubes was used, where $2.5 \times 10^{5}$ tumor cells / $\mathrm{ml}$ were suspended in phosphate buffered saline (PBS). Then 25, 50 and $100 \mu \mathrm{g} / \mathrm{ml}$ from tested compound were added to the suspension, kept at $37{ }^{\circ} \mathrm{C}$ for $2 \mathrm{~h}$. Trypan blue dye exclusion test was then carried out to calculate the percentage of nonviable cells. Doxorubicin (Adriablastina) was used as a positive control. The percentage of the nonviable cells was calculated by the following equation:

$\%$ of non-viable cells $=100\left(\mathrm{~N} / \mathrm{N}_{\mathrm{t}}\right)$, where $\mathrm{N}$ is the number of non-viable cells counted, $\mathrm{N}_{\mathrm{t}}$ is the total number of cells. The test was repeated four times for each compound. The results are summarized in Table 2.

\section{References}

1. Habibi, M. H.; Hassanzadeh, A.; Isfahani, A. Z. Dyes and Pigments 2006, 69, 93.

2. Stefancich, G.; Silvestri, R.; Retico, A.; Artico, M.; Simonetti, G. Arch. Pharmzie 1992, $325,199$.

3. Bhuiyan, M. M. H.; Rahman, K. M. M.; Hossain, M. K.; Rahim, A.; Hossain, M. I.; Abu Naser, M. Acta Pharm. 2006, 56, 441.

4. Fujii, K.; Tsutsumiuchi, K.; Yamanaka,Y.; Nakamura, K. Jpn. Pat. 1999, 11116555; Chem. Abstr. 130, 325151w.

5. Zhang, H. Z.; Kasibhatla, S.; Kuemmerle, J.; Kemnitzer, W.; Ollis-Mason, K.; Qiu, L.; Crogan-Grundy, C.; Tseng, B.; Drewe, J.; Cai, S. X. J. Med. Chem. 2005, 48, 5215.

6. El-Feky, S. A.; Abdel-Samii, Z. K. Pharmazie 1995, 50, 341.

7. Nanteuil, G. D.; Herve, Y.; Duault, J.; Espeinal, J.; Boulanger,M.; Ravel, D. ArzneimForsch Drug Res. 1995, 45, 1175.

8. Wolfbeis, O. S. Monatsh. Fur Chem. 1981, 112, 875.

9. Baraldi, P. G.; PCT Int. Appl. WO. 1999, 21, 617, Chem. Abstr. 130, 296427h.

10. El-Ahl, A. A. S. Phosphorus, Sulfur and Silicon 2000, 156, 189.

11. (a) Shawali, A. S.; Farghaly;T. A. Tetrahedron, 2009, 65, 644. (b) Shawali, A. S.; Mosselhi, M. N.; Farghaly; T. A. J. Chem. Res., 2007, 479. (c) Shawali, A. S.; Sherif, S. M.; Farghaly, 
T. A.; Shehata, M. R.; Darwish, M. A. A. J. Chem. Res. 2007, 44. (d) Shawali, A. S.; Harb, N. M. S.; Badahdah, K. O. J. Heterocycl. Chem. 1985, 22, 1397.

12. Jones, R.; Ryan, A. J.; Sternhell, S.; Wright, S. E. Tetrahedron 1963, 19, 1497.

13. Johnson, C. D.; The Hammett equation, Cambridge University press. 1973, pp 1-24.

14. Humffray, A. A.; Ryan, J. J. J. Chem. Soc. (B) 1969, 1138.

15. Shawali, A. S.; Rizk, M. S.; Abdelhamid, A. O.; Abdallah, M. A.; Parkanyi, C.;Wojciechowska,M. E. Heterocycles 1979, 36, 179.

16. Elhilaly, A. E.; Shawali, A. S.; Madkour, M. A. Inorg. Chim. Acta. 1979, 36, 179.

17. Hassaneen, H. M. Synthetic Comm. 2007, 37, 3578.

18. Kuzueva, O. G.; Burgart, Ya. V.; Saloutin, V. I. Russ. Chem. Bull. 1998, 47, 673

19. Mitchell, A.; Nonhebel, D. C. Tetrahedron 1979, 35, 2013.

20. Van Uitert, L. G.; Haas, C. G. J. Am. Chem. Soc. 1953, 75, 451.

21. Albert, A.; Serjeant, E. P.; The Determination of Ionization Constant of acid, Chapman and Hall Ltd., London 1971, pp 9-39.

22. Gans, P.; Sabatini, A.; Vacca, A. Inorg. Chim. Acta 1976, 18, 237.

23. Mclimans, W. F.; Davis, E. V.; Glover, F. L.; Rake, G. W. J. Immunol. 1957, 79, 428. 